\title{
Importancia de la Sabiduría Didáctica Práctica como Fuente de Conocimiento Base para la Enseñanza de la Anatomía
}

\author{
Importance of Teaching Practical Wisdom as a Source \\ of Knowledge Base for Teaching Anatomy
}

\author{
"Mario Pellón Arcaya; ** Juan Mansilla Sepúlveda \& ${ }^{* * *}$ Daniel San Martín Cantero
}

\begin{abstract}
PELLÓN, A. M.; MANSILLA, S. J. \& SAN MARTíN, C. D. Importancia de la sabiduría didáctica práctica como fuente de conocimiento base para la enseñanza de la anatomía. Int. J. Morphol., 28(1):219-226, 2010.

RESUMEN: La sabiduría práctica es uno de los conocimientos base que se propone para generar prácticas de enseñanza de calidad en el campo de la medicina. Se planteó un diseño metodológico cualitativo - comprehensivo con el propósito de describir la importancia de la sabiduría didáctica práctica como fuente de conocimiento base para la enseñanza de la anatomía en docentes de la Universidad Mayor de Temuco, esto al amparo de la teoría fundamentada, el método comparativo constante y los principios de triangulación y convergencia. La muestra se constituyó con la totalidad de los docentes de la cátedra de Anatomía. Para el análisis de datos se consideró la codificación abierta, de la cual emergieron dos categorías: a) desempeño experto y b) principios de la sabiduría práctica. Las técnicas de recolección de información fueron: observación etnográfica, entrevista semiestructurada y grupos focales. Para el procesamiento de data verbal se utilizó el software para el análisis cualitativo de datos Atlas-ti versión 5.0. Los resultados del estudio develan que la sabiduría didáctica práctica es una fuente de conocimiento relevante en toda práctica pedagógica, valorizándose como el principal conocimiento base para la formación en medicina, en consecuencia se desafía a los profesionales de la enseñanza de la medicina a realizar un tránsito desde el nivel de profesionalidad de principiante hacia un nivel de profesor experto.
\end{abstract}

PALABRAS CLAVE: Anatomía; Enseñanza.

\section{INTRODUCCIÓN}

Las prácticas pedagógicas en el contexto actual de la educación superior deben responder a exigencias de distintos campos: (a) institucional; incluye la dimensión curricular propia del modelo educativo en que se sitúa la práctica de enseñanza, (b) la sociedad de mercado; incluye las competencias de egreso que deben ser formadas desde la enseñanza, para luego ser movilizadas en la profesión, (c) matriz disciplinar; comprende aquellos conocimientos sustantivos que articulan el saber sabio de cada materia.

Todas las disciplinas que se enseñan en la universidad poseen lo que Shulman (1986) denomina un "conocimiento base para la enseñanza": esto es, un conjunto codificado o codificable de conocimientos, destrezas, comprensión y tecnología, ética y disposición, responsabilidad co- lectiva. Para dar cuenta de este conocimiento base cobra sentido la propuesta de Shulman, quien ha sistematizado el conocimiento base en las siguientes categorías: (1) Conocimiento del contenido, (2) Conocimiento didáctico general (principios y estrategias generales de manejo y organización de la enseñanza), (3) Conocimiento del currículo (especial manejo de los materiales y artefactos que sirven como "herramientas para el oficio y el arte" de la enseñanza, (4) Conocimiento didáctico del contenido (amalgama entre materia y pedagogía: esfera exclusiva de los maestros, su forma especial de comprensión profesional), (5) Conocimiento de los alumnos y de sus características (6) Conocimiento de los contextos educativos (7) Conocimiento de los objetivos, finalidades y valores educativos, de sus fundamentos filosóficos e históricos.

\footnotetext{
Médico Cirujano Oftalmólogo. Magíster en Pedagogía Universitaria. Profesor Asistente Facultad de Medicina. Universidad de La Frontera y Universidad Mayor, Temuco, Chile

** Profesor de Estado. Magíster en Desarrollo Regional y Local. Docente Postgrado Universidad Mayor y Universidad Católica de Temuco, Temuco, Chile.

**** Profesor de Estado. Docente Universidad Católica de Temuco, Temuco, Chile.
} 


\section{Fuentes de Conocimiento Base para la Enseñanza}

En 1990 las categorías enunciadas son redefinidas por Grossman et al. (2005) en cuatro áreas generales: conocimiento pedagógico general, conocimiento del contenido, conocimiento pedagógico del contenido y el conocimiento del contexto. En este sentido existen por lo menos cuatro bases de conocimiento: (1) Formación académica en la disciplina a enseñar, (2) materiales y contexto del proceso educativo institucionalizado, (3) investigación sobre escolarización, aprendizaje humano y desarrollo del fenómeno sociocultural del quehacer de los profesores y (4) sabiduría que otorga la práctica misma.

a. Formación académica en la disciplina a enseñar. La primera fuente del conocimiento base es el conocimiento de los contenidos: el saber, comprensión, habilidades y disposiciones que deben adquirir los estudiantes. Este conocimiento se apoya en dos bases: la bibliografía y estudios acumulados en cada una de las disciplinas; y el saber académico histórico-filosófico sobre la naturaleza del conocimiento en estos campos de estudio. Un profesor no solo debe comprender las estructuras de la materia enseñada y los principios de la organización conceptual, sino también debe conocer los principios de indagación que ayudan a responder dos tipos de preguntas: ¿cuáles son, en este ámbito del saber, las ideas y las destrezas más importantes?, y ¿de qué manera quienes generan conocimientos en esta área incorporan las nuevas ideas y descartan las antiguas?. En definitiva, ¿cuáles son las reglas y procedimientos de un buen saber académico y de investigación? Estas interrogantes se clarifican a partir de los aportes de Schwab (1964), quien clasificó el conocimiento de la enseñanza en: conocimiento de estructuras sustantivas y sintácticas, respectivamente. Las fuentes del conocimiento de los contenidos de la asignatura implican necesariamente que el profesor no sólo debe comprender a fondo la materia específica que enseña, sino además debe poseer una amplia formación humanista que otorgue un marco para el aprendizaje adquirido previamente (conocimientos previos), como un mecanismo que facilita la adquisición de una nueva comprensión (aprendizaje significativo). Los docentes tienen una especial responsabilidad respecto al conocimiento de los contenidos de la asignatura, por ser la principal fuente de la comprensión de la materia para los alumnos. La manera en la que esta comprensión se comunica transmite a los estudiantes qué es esencial en una materia y qué es periférico. Frente a la diversidad de sus alumnos, el docente debe tener una comprensión flexible y polifacética, que permita impartir explicaciones alternativas de los mismos conceptos o principios. Los profesores también comunican, conscientemente o no, ideas acerca de las maneras de obtener el conocimiento en un campo, además de una serie de actitudes y valores que influyen notablemente en la comprensión de sus alumnos. Esta responsabilidad exige una profunda comprensión de las estructuras de la materia por parte del profesor, referido a las actitudes y entusiasmo del docente frente al objeto de enseñanza. Por tanto, estos aspectos del conocimiento de los contenidos se entienden como una característica fundamental del conocimiento base para la enseñanza.

b. Estructuras y materiales didácticos. Con el objeto de promover los objetivos de la escolarización organizada, se diseñan materiales y estructuras para la enseñanza y el aprendizaje. Dado que los profesores inevitablemente actúan dentro de una matriz formada por estos elementos, utilizándolos y siendo utilizados por ellos, es lógico que los principios, políticas y circunstancias de su funcionamiento configuren una importante fuente del conocimiento base. No es preciso sostener que esta fuente esté respaldada por una bibliografía específica, aunque existe un amplio conjunto de obras de investigación en la mayoría de estosámbitos. Pero si un profesor tiene que "conocer el territorio" de la enseñanza, entonces debe estar familiarizado con el paisaje compuesto de tales materiales, instituciones, organizaciones y mecanismos. Esto se constituye en las herramientas del oficio y circunstancias contextuales, que facilitarán o inhibirán las iniciativas de enseñanza.

c. Literatura educativa especializada. Una tercera fuente es el importante y creciente caudal de bibliografía académica dedicada a la comprensión de los procesos de escolarización, enseñanza y aprendizaje. En estas obras se incluyen las conclusiones y los métodos de investigación empírica en las áreas de docencia, aprendizaje y desarrollo humano, así como también los fundamentos normativos, filosóficos y éticos de la educación. Los aspectos normativos y teóricos de los conocimientos académicos sobre la enseñanza son tal vez los más importantes. Así y todo, si bien estas conclusiones de las investigaciones son relevantes y merecen ser objeto de un estudio exhaustivo, representan sólo una faceta de la aportación del mundo académico, cuyas influencias más perdurables y poderosas sobre los profesores son probablemente las que enriquecen la imagen que ellos se forman de lo que es posible anhelar: sus visiones de lo que constituye una buena educación, o de cómo se desenvolvería un alumno bien educado si se le ofrecieran oportunidades y estímulos adecuados. Platón, Dewey, Russeau, Skinner \& Freire en sus tesis expresan el ideal de un buen sistema educativo, quienes forman parte de la tradición reflexiva sobre educación. Asimismo, muchos trabajos cuyo propósito es difundir los resultados de investigaciones empíricas, sirven también como importantes fuentes para la enseñanza. En este contexto destacan estudios como los de Bloom (1976) sobre el aprendizaje para el dominio, y de Rosenthal \& Jacobson (1968), Piaget (1969), y epistemo- 
logía genética y psicología evolutiva, Vigostky (1934) con el enfoque socio-histórico cultural, Morín (1998) y el paradigma de la complejidad, Engeström (1987) Teoría de la Actividad y Aprendizaje Expansivo. Independientemente de si las afirmaciones empíricas formuladas por estos autores pueden respaldarse, resulta innegable su impacto en las concepciones de los profesores sobre las finalidades posibles y deseables de la educación. Así pues, las obras de índole filosófica, crítica y empírica que pueden informar los objetivos, las visiones y los sueños de los profesores son un componente esencial del conocimiento base académico para la enseñanza.

Un tipo de conocimiento académico citado con mayor frecuencia se deriva del estudio empírico sobre la enseñanza efectiva. Esta investigación ha sido resumida recientemente por Gage (1978, 1986), Shulman (1986a), Brophy \& Good (1986), y Rosenshine \& Stevens (1986). El objetivo esencial de ese programa de investigación ha sido identificar aquellos comportamientos y estrategias del profesorado que, con mayor probabilidad, van a suscitar un progreso en el rendimiento académico de los alumnos. En otras palabras, los principios de la enseñanza efectiva tienen que ver con el hecho de transformar las salas de clase en lugares donde los alumnos puedan abocarse a tareas de aprendizaje, orientarse hacia el aprendizaje con un mínimo de interrupción y distracción, y recibir una oportunidad equitativa y adecuada para aprender.

d. La sabiduría adquirida con la práctica. La fuente última para el conocimiento base es la menos codificada de todas (Shulman, 1986). Se trata de la sabiduría que se obtie- ne de la práctica misma, las máximas que guían la práctica de los profesores competentes (o proporcionan la racionalización reflexiva para ella). Una de las tareas más importantes para la investigación educativa consiste en trabajar junto con los educadores para desarrollar representaciones codificadas de la sabiduría didáctica práctica de los profesores competentes. Gran parte de la concepción de la enseñanza deriva de la labor de recopilar, analizar y comenzar a codificar la sabiduría que va surgiendo de la práctica de los profesores inexpertos y con experiencia.

Respecto de las evidencias empíricas y discusión teóricas surge el siguiente problema de investigación: ¿Cuál es la importancia otorgada a la sabiduría didáctica práctica como fuente de conocimiento base utilizado por los docentes de anatomía en sus prácticas de enseñanza en la Universidad Mayor de Temuco?.

El objetivo de esta investigación es describir la importancia de la sabiduría didáctica práctica como fuente de conocimiento base para la enseñanza de la anatomía en docentes de la Universidad Mayor de Temuco.

A partir de los resultados de la investigación se propone: codificar los significados que le atribuyen los docentes de anatomía a la sabiduría didáctica práctica como fuente de conocimiento de base. Esto con el propósito de promover líneas investigativas que den cuenta del saber pedagógico en el campo de la enseñanza de la medicina, cuyas implicancias se dirigen al develamiento de las categorías constituyentes de un profesor experto en el contexto de la enseñanza de la anatomía humana.

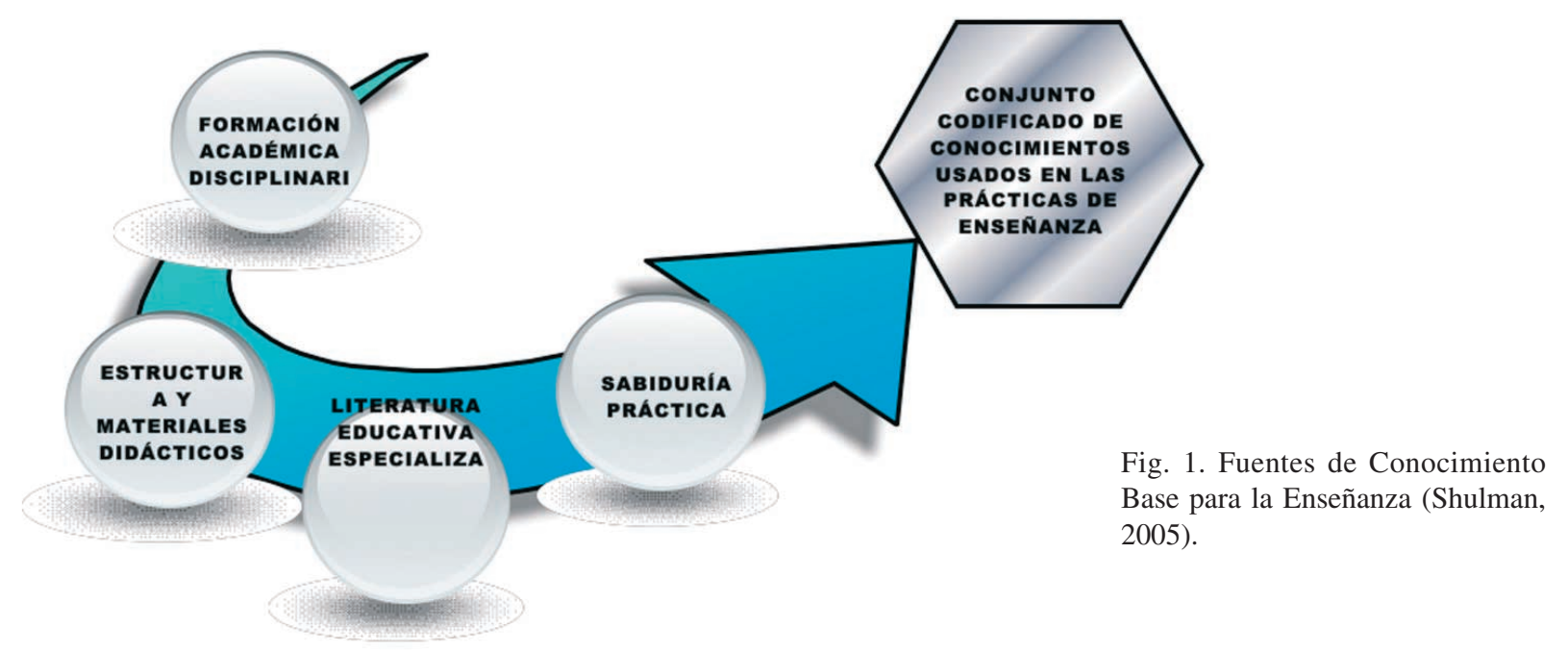




\section{METODOLOGÍA}

Para este estudio se ha considerado el método cualitativo situado en un paradigma hermenéutico con alcances descriptivos donde los significados intersubjetivos están situados y construidos en el marco de la vida social sin distorsionarla y someterla a controles experimentales, eligiendo la descripción espesa y los conceptos comprensivos del lenguaje simbólico (Ruiz, 2003, Flick, 2004 \& Geertz 1983).

La muestra fue intencional, no probabilística y la constituyeron todos los docentes de la cátedra de Anatomía de la Escuela de Medicina de la Universidad Mayor, quienes imparten docencia a 70 estudiantes de primer año. Las técnicas de recolección de datos fueron entrevistas semiestructuradas, grupos focales y observaciones etnográficas.

Los grupos focales o entrevista de grupo son "aquellas en las que un número de personas son reunidas en un emplazamiento o lugar para que expresen sus opiniones, revelen sus actitudes o manifiesten sus conductas" (Ruiz, 2003, p. 248-249). Así, uno de sus propósitos es permitir tanto la homogeneidad de opiniones y disparidades de criterios. A su vez, la entrevista contribuye a la manifestación espontánea de significados y jerarquías de valores del entrevistado (Ruiz, 2003).

En la etapa de "saturación teórica" se desarrolló el muestreo teórico donde los datos se tornaron repetitivos. Flick define el criterio de 'saturación teórica' de la siguiente manera: “(...) el muestreo e integración de material nuevo se acaba cuando la "saturación teórica" de una categoría o grupo de casos se ha alcanzado, es decir, cuando no emerge ya nada nuevo" (Flick, 2004, p. 79).
Al respecto Strauss \& Corbin (2002) señalan que el criterio que asegura la riqueza y densidad explicativa, y de relaciones teóricas entre categorías es la saturación teórica de éstas, entonces el único criterio para finalizar el proceso de recolección de datos es la saturación de los mismos, esto finalmente explicará la variabilidad de las categorías.

En esta investigación se realizó una codificación abierta, proceso en que el investigador aborda el texto, con el fin de develar conceptos, ideas y sentidos. Respecto a esto Strauss y Corbin (2002) señalan: “...que para descubrir y desarrollar los conceptos debemos abrir el texto y exponer los pensamientos, ideas y significados contenidos en él." (Strauss \& Corbin, 2002, p. 111). Durante este proceso de codificación abierta los datos fueron descompuestos, examinados y comparados en términos de sus similitudes y diferencias, al amparo de una aproximación inductiva a la realidad, por lo que no precisa de una teoría para aplicar sus conceptos, leyes o dimensiones en el texto que se está codificando; por el contrario, el investigador debe zambullirse en el texto para así identificar los conceptos, dimensiones y construir categorías y subcategorías pertinentes a la investigación.

\section{RESULTADOS}

Una vez realizada la codificación abierta de datos verbales, se constituye la categoría sabiduría didáctica práctica, a partir de la cual se desprenden dos subcategorías: (1) desempeño experto y (2) principios de la sabiduría práctica. La subcategoría desempeño experto comprende el código exigencia de la actualización profesional, por otra parte para la subcategoría principios de la sabiduría práctica resultaron los siguientes códigos: actuación prospectiva en la práctica y transferabilidad del conocimiento conceptual (Fig. 2).

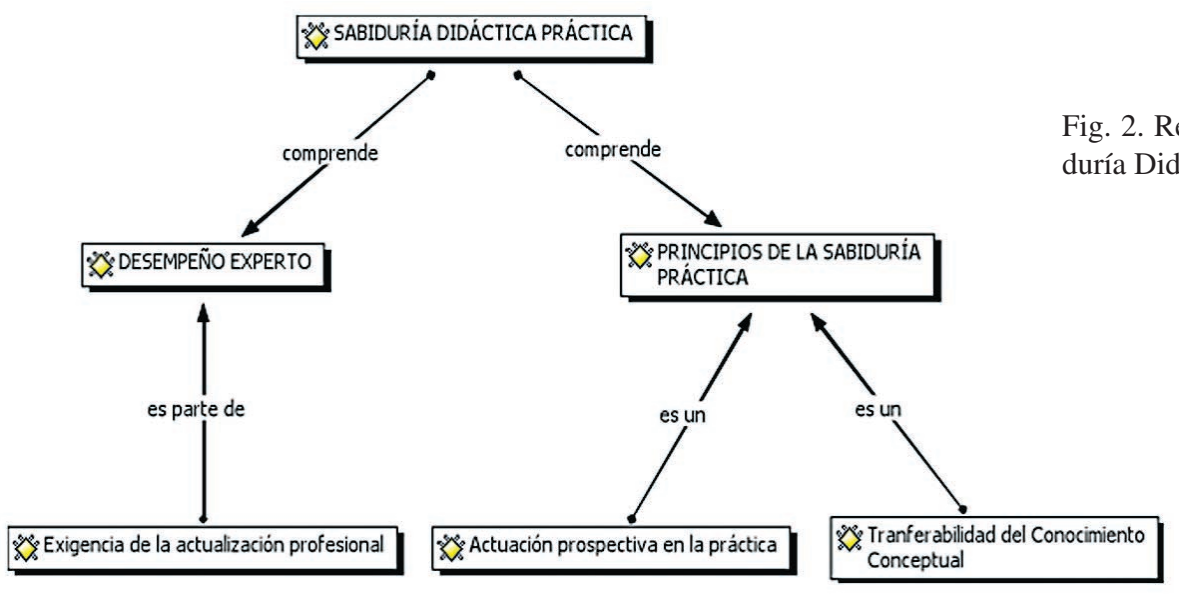




\section{Categoría Sabiduría Didáctica Práctica.}

Consiste en aquella sabiduría que se configura y valida en la práctica misma, asimismo se entiende como una competencia del profesional que se evidencia en la toma de decisiones responsablemente, a partir de la movilización de sus saberes teóricos hacia el campo procedimental y actitudinal.

\section{a) Subcategoría Desempeño Experto.}

En esta subcategoría los participantes explicitan la necesidad de la actualización profesional, debido a las nuevas evidencias empíricas que han afectado al objeto de saber propio de la anatomía. Esto afecta a las prácticas de enseñanza en sus dimensiones conceptuales y metodológicas: “... debe estar al día, la malla o el curriculum de la carrera no puede ser el mismo de veinte años atrás, porque las realidades eran diferentes, los antecedente epidemiológicos han cambiado, entonces la OMS habla mucho de educación médica y la educación médica también está relacionada no solamente con temas sino también con la metodología" (Entrevista 03 [125:125]). En este sentido, lo anterior forma parte de una de las fuentes base para la enseñanza, así posteriormente conformará el sustento conceptual necesario para el desarrollo de la sabiduría didáctica práctica.

\section{Principios de la Sabiduría Práctica}

La actuación prospectiva de la práctica es un aspecto relevante para la sabiduría didáctica práctica, de esta manera el informante clave manifiesta la importancia de reconocer los fines últimos de los saberes insertos en la formación profesional: “... entonces en la trascendencia de aquello, o sea siempre en nuestra asignatura es que el alumno tenga claro cuál es la proyección, que no es un saber por saber, es un saber concreto y aplicable, tal como la ducha y el perfume..." (Entrevista 01 [254:254]). Esto hace referencia a la capacidad de racionalización reflexiva del docente para la práctica, poniendo en evidencia estrategias globales de metaconocimientos.

Un último constructo codificado corresponde a la transferibilidad de conocimientos, esto quiere decir que el profesor que posee la sabiduría didáctica práctica es capaz de transferir de manera coherente y estructurada la dimensión declarativa formal del saber a una dimensión práctica sustantiva del saber: “... sí podría caer en eso, pero en la esencia de la asignatura lo fundamental es la práctica, que apliquen en una estructura tridimensional los conocimientos que impliquen, los conocimientos que uno ha tratado de entregarle al alumno, así también viendo o yéndose demasiado a lo teórico no vamos a tener después alguien que pueda practicar lo que es la medicina, sino que sólo pueda escribir de..." (Entrevista 02 [257:257]). Así el informante indica que es necesario considerar la práctica para la transposición del objeto del saber disciplinario.

\section{DISCUSIÓN}

En el estudio realizado, cuyo objetivo general describir la importancia de la sabiduría didáctica práctica como fuente de conocimiento base para la enseñanza de la anatomía en docentes de la Universidad Mayor de Temuco, es posible señalar que los docentes reconocen como relevante la actualización profesional, la actuación prospectiva y la transferabilidad del conocimiento.

Respecto de la actualización profesional, Shulman (1986) la considera como tercera fuente del conocimiento base para la enseñanza, bajo la denominación de literatura educativa especializada. Esto supone una exigencia para la concreción de la sabiduría práctica. Lo anterior, de acuerdo a Huberman (1985), es un rasgo esencial de un profesor experto, esto lo ratifica Leinhardt (1990) planteando que un profesor experimentado posee un "conocimiento práctico de su profesión", o "sabiduría de la práctica". Así un docente experimentado busca fuera de la clase la información y la destreza que le pueda aportar la solución de un problema en la práctica diaria.

En cuanto a la actuación prospectiva de la práctica es pertinente citar a Ortega en su conocido artículo pedagogía y anacronismo, en donde se refiere a la aportación del pedagogo alemán Georges Kerschensteiner (1965), quien analiza los fundamentos filosóficos de la pedagogía y concluye:

“... con lo cual venimos a la grotesca situación de que los niños de 1940 son educados conforme a las ideas y conocimientos de 1890, y que la escuela cuya pretensión es precisamente organizar el porvenir, vive de continuo retrasada dos generaciones..." (p. 81)

Lo anterior expresa la idea de que las características del sistema educativo operan instrumentalmente como reproductores de los saberes disciplinarios y no como sistemas dinámicos en el campo profesional de la medicina, esto exige una presencia discursiva y metodológica de una pedagogía prospectiva. Sobretodo hoy donde el quehacer docente está expuesto al dinamismo propio de la sociedad del conocimiento (Castell, 2001).

Finalmente, la transferabilidad del conocimiento se 
constituye en un código relevante, que explica la necesidad de transferir el saber conceptual a un saber aplicado para resolver situaciones complejas en la práctica del ejercicio médico: “... supone el reconocimiento de una identidad de estructura entre los problemas y situaciones, exige gran repertorio de soluciones para situaciones variadas" (Le Boterf, p. 113). Al respecto, es preciso enfatizar que los saberes son difícilmente transferibles en si mismos, o sea, no hay, - por naturaleza -, saberes que sean transferibles y otros que no lo sean. Entonces el carácter de transferible no hay que buscarlo "en los conocimientos", sino en la sabiduría que otorga la práctica, pues ésta predispone "la facultad de transferir". Se han planteado tres dimensiones relevantes de la sabiduría didáctica práctica, que se manifiestan en el discurso de los docentes de la asignatura de anatomía. Sin embargo, es necesario clarificar que los elementos discutidos no constituyen la totalidad de las dimensiones que subyacen a la sabiduría didáctica práctica propiamente tal. Es por esto que se abren nuevas posibilidades de investigación (Fig. 4).

\section{CONCLUSIONES}

A partir de este estudio es posible constatar que la sabiduría didáctica práctica es una fuente de conocimiento relevante en toda práctica pedagógica. De esta manera es necesario valorizarla como el principal conocimiento base para la formación en medicina. Para esto se requieren profesionales de la enseñanza de la medicina que se trasladen desde el nivel de profesionalidad de principiante hacia un nivel experto (Le Boterf, 2000). Esto implica, entre otros aspectos: gestionar modos de intervención que movilicen en la docencia saberes disciplinarios hacia saberes prácticos, contextualización del saber, desarrollo de meta-conocimientos, gestión del tiempo, grados de autonomía, cono- cimientos de los puntos fuertes y los límites de su pericia y una regularidad muy grande de las competencias.

En este contexto la discusión metodológico-disciplinaria y las evidencias empíricas entorno a la sabiduría didáctica práctica en la enseñanza de la medicina son escasas. En este sentido las tesis de Shulman (1986, 1990 \& 2005) se constituyen en un marco explicativo teórico-formal relevante para comprender desde el conocimiento base la sabiduría didáctica práctica en la docencia del conocimiento específico de las materias de anatomía.

Precisamente los docentes de anatomía manifiestan algunos rasgos de la sabiduría didáctica práctica que se explicitan regularmente en sus discursos, tales como: actualización profesional, actuación prospectiva en la práctica y transferabilidad del conocimiento conceptual. Sin embargo, no se visibilizan todas las dimensiones que constituyen la sabiduría didáctica práctica (Fig. 3)

Los actuales modelos pedagógicos de formación en la educación superior, específicamente aquellos orientados a la formación basada en competencias, demandan formas de actuación y aprendizaje situados en la práctica misma (OCDE, 2009), por tanto la enseñanza debe nutrirse con elementos de la sabiduría didáctica práctica.

Finalmente, se sugiere la necesidad de codificar la dimensión de la sabiduría didáctica práctica a partir de la enseñanza presente en docentes de anatomía, con el propósito de develar la relación dialéctica existente entre el saber teórico y el saber práctico, y en este último comprender la díada saber-hacer/ saber-actuar representa un desafío didáctico disciplinario fundamental en la docencia de la medicina.

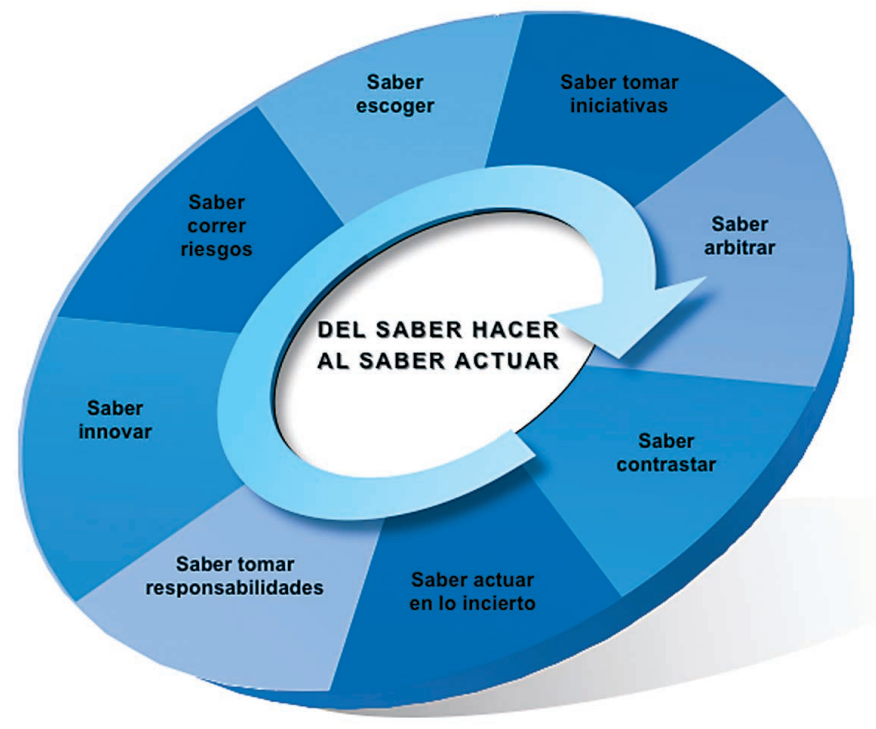

Fig. 3. Sabiduría práctica en la complejidad de la situación profesional. Elaboración propia adaptado de Le Boterf (2000). 
PELLÓN, A. M.; MANSILLA, S. J. \& SAN MARTíN, C. D. Importance of teaching practical wisdom as a source of knowledge base for teaching anatomy. Int. J. Morphol., 28(1):219-226, 2010.

SUMMARY: Practical wisdom is one of the proposed base knowledge to generate best practices for teachings in the field of medicine. A methodological qualitative-comprehensive design was proposed with the purpose of describing the importance of the didactical practical wisdom as source of base knowledge for the teaching of anatomy by the faculty of the Universidad Mayor of Temuco, this was done based on the founded theory, constant comparative method and principles of triangulation and convergence. The sample was composed of the entire faculty team for the anatomy course. For the analysis of the data, open codification was considered, from which two categories surfaced: a) expert performance and b) principles of practical wisdom. The techniques for data gathering were: ethnographic observation, semi structured interview and focus groups. The processing of the verbal data was done by means of a software for qualitative analysis, Atlas-ti version 5.0. The results of the study show that didactical practical wisdom is a source of relevant knowledge in all pedagogical practice, assessing it as the principal base knowledge for the training in medicine, therefore a challenge is made to all professionals involved in teaching medicine to transition from basic professionalism into an expert one.

KEY WORDS: Anatomy; Teaching.

\section{REFERENCIAS BIBLIOGRÁFICAS}

APA. American Psychology Association. Manual de Estilos de Publicaciones de la American Phychological Association: guía de entrenamiento para el estudiante. Bogotá. El Manual Moderno, 2006.

Castells, M. Era de la Información. 2 Ed. Madrid, Alianza Editorial, 2001.

Dewey, J. Experience and Education. London \& New York, Macmillan, 1938.

Engeström, Yrgo. Learning by Expanding. An ActivityTheoretical Approach to Developmental Research, Orienta Konsultit Oy, Helsinki, 1987.

Flick, U. Introducción a la investigación cualitativa. Madrid, Ediciones Morata, 2004.

Freire, P. La educación como práctica de la libertad. México, Siglo XXI, 1980.

Grossman, P.; Wilson, S.; Shulman, L. . Profesores de sustancia: el conocimiento de la materia para la enseñanza. Rev. Revista de currículum y formación del profesorado, 9:2-25, 2005.

Le Boterf, G. Ingeniería de las competencias. $1^{\circ}$ Ed. Barcelona, Gestión 2000 S.A, 2001.

Leinhardt, G. Capturing craft knowledge in teaching. Educational Researcher, 19(2):18-25, 1990.

Medina, A. De la experiencia profesional a la sabiduría didáctica. Rev. Enseñanza 23:269-85, 2005.
Morin, E. Los siete saberes necesarios para la educación de futuro. UNESCO, 1999.

OCED. La educación superior en Chile. Revisión de Políticas Nacionales de Educación. Ministerio de Educación de Chile, Chile, 2009.

Ortega y Gasset J. Misión de la Universidad y otros ensayos a fines. 3 Ed. Madrid, Revista de Occidente, 1930.

Piaget, J. Psychology of Intelligence. Paterson, NJ. Littlefield Adams, 1954.

Platón. Diálogos. Madrid, Gredos, 1993.

Rosenthal, R. \& Jacobson, L. Pygmalion in the Classroom. New Cork. Holt, Rinehart \& Winston, 1968.

Ruiz, J. Metodología de la Investigación Cualitativa. Bilbao. Universidad de Deusto, 1996.

Shulman, L. Conocimiento y enseñanza: fundamentos de la nueva reforma. (Knowledge and Teaching: Foundations of the New Reform) Stanford University Revista de currículum y formación del profesorado, 2005.

Skinner, B. F. The operational analysis of psychological terms. In: A. C. Catania \& S. Harnad (Eds.), The selection of behavior: The operant behaviorism of B. F. Skinner. Comments and consequences. New York. Cambridge University Press, 1988c. pp.150-217.

Strauss, A. \& Corbin, J. Bases de la investigación cualitativa. Técnicas y procedimientos para desarrollar la teo- 
PELLÓN, A. M.; MANSILLA, S. J. \& SAN MARTíN, C. D. Importancia de la sabiduría didáctica práctica como fuente de conocimiento base para la enseñanza de la anatomía. Int. J. Morphol., 28(1):219-226, 2010.

ría fundamentada. Medellín-Colombia. Universidad de Antioquia, 2002.

Schwab, J. J. The structure of the disciplines:Meanings and significances. En G.W. Ford y L. Pugno (eds.), The structure of knowledge and the curriculum. Chicago, Rand Mc Nally, 6-30, 1964.

Vigotsky, L. S. Mind in society. The development of higher psychological processes. Cambridge, M. A. Harvard University Press, 1994.
Dirección para correspondencia:

Mario Pellón Arcaya

Médico Cirujano Oftalmólogo.

Magíster en Pedagogía Universitaria.

Profesor Asistente Facultad de Medicina. Universidad de La Frontera y Universidad Mayor

Casilla 54-D, Temuco

CHILE

Email: mpellon@ufro.cl

Recibido : 23-12-2009

Aceptado: 16-01-2010 